\title{
Radiographic predictors of deterioration in patients with lumbosacral lipomas
}

\author{
Albert Tu, MD, FRCSC, ${ }^{1}$ Alexander R. Hengel, BSc, ${ }^{2}$ and D. Douglas Cochrane, MD, FRCSC ${ }^{2}$ \\ 'Division of Neurosurgery, Vancouver General Hospital; and 2Division of Pediatric Neurosurgery, British Columbia Children's \\ Hospital, University of British Columbia, Vancouver, British Columbia, Canada
}

\begin{abstract}
OBJECTIVE Although patients with lumbosacral lipomas may be asymptomatic at presentation, most develop neurological symptoms over time. Given the challenges in examining infants, the authors sought to determine whether MRI would be helpful in identifying patients who are more likely to deteriorate early in life and who would potentially benefit from early surgical intervention.

METHODS A retrospective review of all patients with lumbosacral lipomas who were seen at the authors' institution between 1997 and 2013 and who were managed without prophylactic surgery was performed. The clinical history and imaging results for each patient were reviewed in detail and then correlated to the pattern of and age at clinical deterioration.

RESULTS Twenty-four patients were identified. Nine worsened within the first 18 months of life (early deterioration), and 15 patients deteriorated or remained stable after 30 months (late deterioration/stable). No patients worsened between 18 and 30 months of age. Patients who deteriorated early were more likely to have large intradural lipomas that filled the canal, increased during the 1st year of life, and compressed neurological structures. Some had a syrinx extending above the neural-lipoma interface. Syrinxes in patients with early deterioration were large and expanded in infancy. Patients with early deterioration had motor deficits at the time of deterioration, whereas patients with late deterioration developed mixed urological and motor dysfunction.
\end{abstract}

CONCLUSIONS Patients with large lipomas displacing the cord and an enlarging syrinx have a propensity for early clinical deterioration. Given this, their families may be counseled that 1) the risk of deterioration in infancy may be higher than in infants without these features, and 2) they require more diligent observation. Intervention before deterioration in these infants should also be considered. Patients without these features may be safely observed to a lesser extent.

http://thejns.org/doi/abs/10.3171/2016.1.PEDS15614

KEY WORDS conus lipoma; lumbosacral lipoma; lipomyelomeningocele; magnetic resonance imaging; MRI; radiographic predictors; spine

$\mathrm{L}$ UMBOSACRAL lipomas are the most common spinal dysraphism seen at the British Columbia Children's Hospital (BCCH). The clinical presentation is variable and many patients are initially asymptomatic., $3,10,19,23,24$ The natural history of this condition is not well defined, and controversy surrounds its management. ${ }^{10,17,18,24}$ Clinical assessment is further challenging given the presentation at infancy and the presence of congenital neurological deficits. ${ }^{3}$ Magnetic resonance imaging allows the anatomy of the malformation to be characterized.

The BCCH Spinal Cord Clinic (SCC) is the sole clinic to provide comprehensive multidisciplinary assessment and regular follow-up for patients with spinal dysraphism in British Columbia. The clinic provides families of infants with lumbosacral lipoma with the available natural history data and surgical treatment results and offers families 2 management options: 1) prophylactic resection of the lipoma and untethering of the neurological structures, or 2) regular multidisciplinary review with operative intervention when the patient shows indication of a new deficit. In the absence of a family decision, an observational approach is provided. Standardized follow-up is provided to all patients regardless of the timing of a decision to intervene surgically.

During follow-up of the infants in this series, it became apparent that some infants deteriorated during the 1 st year of life. Was the cause of deterioration tethering or could there have been other factors at play? We sought to determine if there were anatomical features evident on MRI done in infancy that would allow prediction of an

ABBREVIATIONS BCCH = British Columbia Children's Hospital; ED = early deterioration; LD/S = late deterioration or stable; SCC = Spinal Cord Clinic.

SUBMITTED October 16, 2015. ACCEPTED January 20, 2016.

INCLUDE WHEN CITING Published online April 15, 2016; DOI: 10.3171/2016.1.PEDS15614. 
infant's clinical course and thus support an individualized approach to treatment.

\section{Methods}

A retrospective review of all patients with lumbosacral lipomas seen at BCCH SCC between 1997 and 2013 was performed. The study population was defined using a prospective database maintained by the $\mathrm{BCCH}$ Division of Neurosurgery and was composed of those patients whose parents elected a nonoperative, monitored approach to initial management. Patients with complete clinical assessments and MRI results were reviewed in detail. Patients undergoing prophylactic surgical intervention (i.e., patients who underwent surgery prior to developing new symptoms related to their malformation) or those with isolated filum lipomas were excluded. Patients were categorized at presentation as having either stable congenital deficit (present at birth and thought to be due to malformation) or a new or evolving deficit. ${ }^{3}$ The patient's age at onset and the nature of the deterioration were recorded.

All patients were initially assessed and monitored by a multidisciplinary team (comprising neurosurgery, pediatrics, urology, physiotherapy, occupational therapy, nursing, and social work). Clinical reviews with renal and bladder ultrasound examinations were performed every 3 months. Urodynamics were performed for baseline evaluation in the 1st year and when clinical change was apparent. ${ }^{8,9}$ All patients underwent baseline MRI within the first 3 months of life. This study was repeated if there was clinical deterioration and later in the series, every 6 months if a syrinx was found on the initial study.

The lipomas were categorized using the Chapman classification and the pathological features of the lipoma as seen on MRI were documented. Specifically, we assessed the number of intradural spinal levels covered by the lipoma, the extent of lipoma within an intact spinal canal, the degree to which the cord was compressed by the lipoma within the intact canal, the most rostral level of the lipoma, the extent of the laminar defect, the presence of a syrinx rostral to the lipoma-cord interface, and whether the syrinx was noted to change in size over time.

All MR images for patients in the study were reviewed independently by 2 trained neurosurgeons who did not have access to the clinical outcomes at the time of the image review. Clinical data, independent of the imaging data, were reviewed and verified by all 3 authors prior to data analysis.

Statistical analysis was performed using SPSS version 21.0 (SPSS, Inc.). The frequency and counts were analyzed using chi-square and Fisher's exact tests, and a p value of 0.05 was considered significant. Nonparametric data were analyzed using the Mann-Whitney U-test.

Ethics approval for this study was obtained from the Children's and Women's Research Ethics Board.

\section{Results}

A total of 58 patients were identified from the $\mathrm{BCCH}$ Division of Neurosurgery's database. Of these, 44 patients were found to have a lumbosacral lipoma with complete radiographic and clinical documentation. Twenty families elected prophylactic detethering and were excluded, leaving 24 patients for this observational study (Fig. 1). Based on the bimodal distribution of patients' ages at the onset of deterioration (Fig. 2), we stratified patients into 2 groups: early deterioration (ED), which comprised patients younger than 18 months of age at the time of deterioration, and late deterioration or stable $(\mathrm{LD} / \mathrm{S})$, which comprised patients who deteriorated after 18 months of age or had no deterioration at last follow-up.

Nine patients (38\%) were classified as ED and 15 (62\%) were classified as LD/S. The ED group comprised 5 boys $(56 \%)$ and the $\mathrm{LD} / \mathrm{S}$ group comprised 6 boys (40\%). Within the LD/S group, 5 patients had more than 28 months (median 80.87 months, range 22.3-207.6 months) of follow-up and remained clinically stable without deterioration. The remaining 10 patients had deterioration after 30 months of follow-up. No patients worsened between 18 and 30 months.

Three patients (33\%) in the ED group were clinically asymptomatic at baseline, as were 8 patients (53\%) in the $\mathrm{LD} / \mathrm{S}$ group $(\mathrm{p}=0.42)$. The pattern of congenital deficits found at initial presentation differed between these groups. Orthopedic (i.e., foot or spinal alignment abnormalities) or neurological (i.e., stable unilateral lower-extremity weakness) anomalies were more common in the ED group, while congenital deficits seen in the $\mathrm{LD} / \mathrm{S}$ patients were urological (i.e., dribbling incontinence or recurrent urinary tract infections with normal urodynamic studies) or orthopedic (Table 1).

The median age at diagnosis of clinical deterioration was 5.9 months in the ED and 60.0 months in the $\mathrm{LD} / \mathrm{S}$ groups (Table 2). In the ED group, worsening motor/sensory function developed in 7 patients (78\%), bladder dysfunction in 5 patients (56\%), and orthopedic dysfunction in 4 patients (44\%) during follow-up (Table 3). In comparison, 9 patients (90\%, excluding those without any decline) in the LD/S group developed voiding dysfunction. Most commonly it took the form of incontinence, and/or urodynamic studies showing a decrease in bladder capacity with partial spastic neurogenic dysfunction.

We identified several radiographic features that were more frequently found in patients in the ED group compared with patients in the LD/S group (Table 4). Eight patients (89\%) in the ED group had a lipoma that caused deviation and compression of neurological structures, and

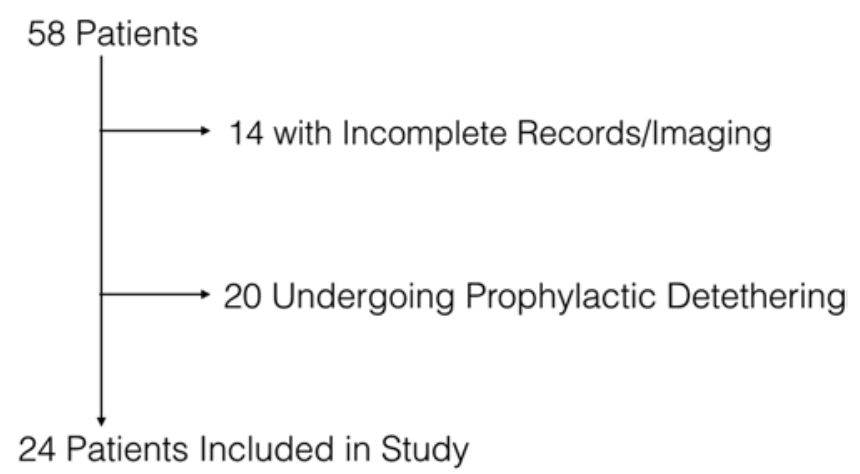

FIG. 1. Patient selection and study population. 


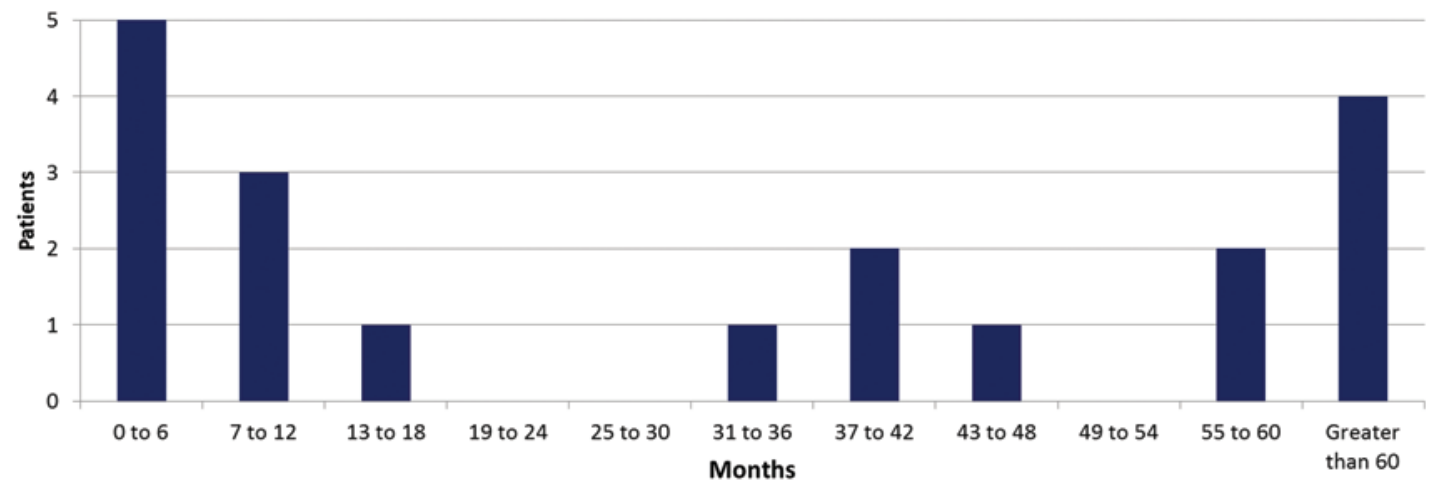

FIG. 2. Bimodal distribution of time to deterioration. Figure is available in color online only.

that filled the canal. Syrinxes were present in 7 patients (78\%). These syrinxes were large and in some patients increased in size during the observation period. Only 1 patient $(7 \%)$ in the LD/S group had deviation of neural structures $(\mathrm{p}<0.01)$ and 4 patients $(27 \%)$ had a fat-filled canal ( $<<0.01$ ) (Fig. 3). Smaller syrinxes were seen in 3 patients (20\%) in the LD/S group (Fig. 4).

Four patients (27\%) in the LD/S group showed a particular appearance of cord and lipoma herniation through the spina bifida, with apparent cord tissue lying in the subcutaneous fat. These lesions did not have an intramedullary cyst or meningocele component. This morphology was only seen in the LD/S group (Fig. 5).

\section{Discussion}

It has been the practice in the $\mathrm{BCCH} \mathrm{SCC}$ to provide families, at the time of diagnosis, with information describing the natural history of lumbosacral lipoma and to offer 1 of 2 management strategies: 1) prophylactic resection of the lipoma and untethering of the neurological structures after imaging and multidisciplinary assessment; or 2) regular multidisciplinary review with operative intervention when the patient shows indication of a new deficit. In the absence of a decision, an observational approach has been provided.

Standardized follow-up is provided to all patients regardless of the timing of their surgical intervention. Parents were counseled about the symptoms that were likely to be due to congenital malformation if present and those symptoms that could indicate deterioration. Specifically, parents were asked to monitor changes in the urinary stream and to report urinary tract infection, changes in foot posture, or new asymmetrical movement. For older

TABLE 1. Comparison of neurological status at presentation in infancy between ED and LD/S groups

\begin{tabular}{lccc}
\hline Presenting Deficit & ED, $n=9(\%)$ & LD/S, $n=15(\%)$ & $p$ Value \\
\hline Asymptomatic & $3(33)$ & $8(53)$ & 0.42 \\
\hline Bladder & $1(11)$ & $4(27)$ & 0.61 \\
\hline Motor/sensory & $3(33)$ & $3(20)$ & 0.63 \\
\hline Orthopedic & $4(44)$ & $5(33)$ & 0.40 \\
\hline
\end{tabular}

children, a standardized timed exercise routine was established and the times monitored.

The assessment of neurological deterioration in infancy is a challenge. Anticipating the infant's developmental potential, while accounting for existing congenital deficits if present, requires a collaborative program of evaluation that includes the patient's family, physiotherapy, and medical specialties. Attentive and educated families are often the first to bring changes to attention. Although these changes do not always indicate deterioration, they serve as an indicator to focus the attention of the multidisciplinary team.

Formal pediatric, orthopedic, urological, neurosurgical, nursing, physiotherapy, and developmental assessments were made quarterly in infancy and annually in childhood. Baseline imaging was performed by 3 months of age. Routine renal ultrasound examinations were done at all visits, with baseline urodynamics at 1 year for asymptomatic, clinically normal patients or at the time of concern about deterioration. Magnetic resonance imaging of the cord and brain was performed at or before 3 months of age and as needed thereafter. Those patients with a syrinx rostral to the lipoma-cord interface underwent MRI every 6 months.

The cause for deterioration in patients with lumbosacral lipomas is not well understood. Many authors have suggested that tethering of the spinal cord by the lipoma leads to injury. ${ }^{2,10,17,18,25}$ Alternatively, patients with large lipomas may experience a direct compressive effect of the lipoma upon the conus and cauda equina. Murata et al. described improvement in a patient with a large lumbosacral lipoma through decompression of the thecal contents without direct untethering, supporting a compressive pathophysiology in at least some patients. ${ }^{15}$ A combination of these factors probably contributes (with different ana-

TABLE 2. Demographic data in 24 patients with lumbosacral lipomas

\begin{tabular}{lccc}
\hline \multicolumn{1}{c}{ Variable } & ED, $n=9$ & LD/S, $n=15$ & $p$ Value \\
\hline Male, no. (\%) & $5(56)$ & $6(40)$ & 1.0 \\
\hline $\begin{array}{l}\text { Asymptomatic at presentation, } \\
\text { no. (\%) }\end{array}$ & $3(33)$ & $8(53)$ & 0.42 \\
\hline Median age at deterioration (mos) & 5.9 & 60.0 & NA \\
\hline NA = not applicable. & & &
\end{tabular}


TABLE 3. New symptoms at the time of clinical deterioration

\begin{tabular}{cccc}
\hline Pattern of Deterioration & ED, $n=9(\%)$ & LD, $n=10(\%)$ & p Value \\
\hline Bladder & $5(56)$ & $9(90)$ & 0.14 \\
\hline Motor/sensory & $7(78)$ & $5(50)$ & 0.35 \\
\hline Orthopedic & $4(44)$ & $3(30)$ & 0.65 \\
\hline
\end{tabular}

tomical configurations of the lipoma, the cord-lipoma interface, and the tissues in the lipoma) to predispose some patients to deterioration.

In our study, patients in the ED group had more expansive lipomas that filled the intact spinal canal while displacing/compressing the conus and cauda equina. These findings suggest that the neurological structures, already compromised, may be unable to accommodate further growth of the lipoma in infancy. Previous studies have shown that there is a rapid deposition of body fat that peaks at 10 months of age and later tapers off. ${ }^{11}$ Given that intraspinal lipomas may behave similarly to other body fat stores, this physiological fat accumulation may explain why some patients deteriorate earlier in infancy, particularly if neurological structures are already impacted by the lipoma and/or are contained within an intact spinal canal. ${ }^{5}$

The presence of a syrinx acting as a mass may be an independent risk factor for further deterioration. An enlarging syrinx may expand the spinal cord and compress the cord-lipoma interface, predisposing the patient to injury.

Whereas some of the features seen on MRI may seem intuitive in predicting early neurological worsening, the presence of dorsal cord herniation is less clear. The patients we observed with this condition have a prototypical extension of their distal spinal cord dorsally through their spina bifida. This structure is encased in a dorsally situated lipoma. There is no cystic dilation within the terminal cord (Fig. 5). This malformation may be comparable to a terminal myelocystocele in which the distal neural

TABLE 4. Radiographic features seen in the ED and LD/S cohorts

\begin{tabular}{lccc}
\hline \multicolumn{1}{c}{ Radiographic Feature } & ED, $n=9$ & LD/S, $n=15$ & $\mathrm{p} \mathrm{Value}$ \\
\hline $\begin{array}{l}\text { Chapman classification } \\
\text { transitional, no. (\%) }\end{array}$ & $9(100)$ & $11(73)$ & $0.26^{*}$ \\
\hline Avg highest spinal level of lipoma & $\mathrm{L}-4$ & $\mathrm{~L}-5$ & $\mathrm{NA}$ \\
\hline $\begin{array}{l}\text { Mean no. of spinal levels covered } \\
\text { by lipoma }\end{array}$ & 5 & 4 & $0.06 \dagger$ \\
\hline $\begin{array}{l}\text { Mean no. of levels of spina bifida } \\
\text { defect }\end{array}$ & 3 & 3 & $0.73 \dagger$ \\
$\begin{array}{l}\text { Lipoma displaces neurological } \\
\text { structures, no. (\%) }\end{array}$ & $8(89)$ & $1(7)$ & $<0.01^{*}$ \\
\hline $\begin{array}{l}\text { Lipoma fills spinal canal, no. (\%) } \\
\text { Dorsal herniation of spinal cord, } \\
\text { no. (\%) }\end{array}$ & $8(89)$ & $4(27)$ & $<0.01^{*}$ \\
\hline Syrinx present, no. (\%) & $7(78)$ & $3(20)$ & $0.26^{*}$ \\
\hline Mean max diameter of syrinx, mm & 6.7 & 1.9 & $0.01^{*}$ \\
\hline Avg avage; max = maximal. & & & \\
\hline
\end{tabular}

Avg $=$ average $;$ max $=$ maximal.

* Chi-square and Fisher's exact test.

$\dagger$ Mann-Whitney U-test.
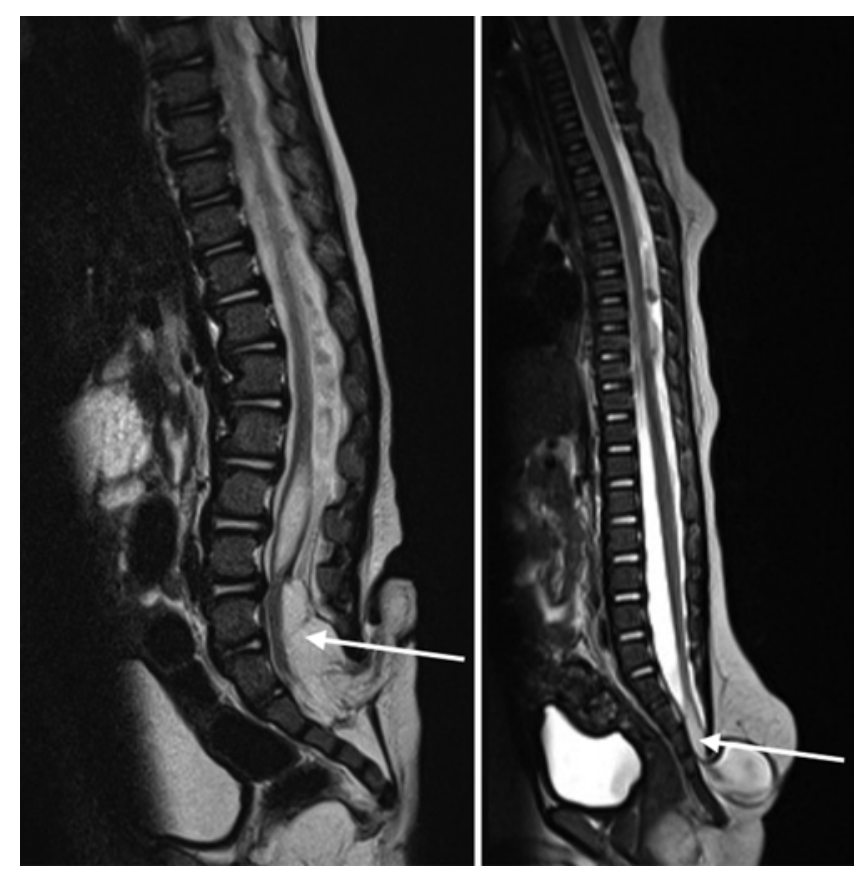

FIG. 3. Lipoma filling the lumbar spinal canal and displacement of neurological structures. Left: The neural placode is displaced to the ventral surface of the spinal canal; the lipoma is contained within an intact spinal canal (arrow). A syrinx extends rostrally in the spinal canal from the lipoma-cord interface. Right: Subarachnoid space is visualized anterior and lateral to the lipoma-cord interface. The conus is elongated and enlarged by intramedullary fat (arrow) but not externally compressed. At surgery, this lesion was found to be a transitional lipoma.

tube herniates dorsally, albeit with a trumpet-like cystic dilation of the terminal structures in addition to other findings. $7,16,21$

Some authors have argued that myelocystoceles warrant early operative intervention given their propensity for precipitous deterioration due to rapid expansion of the cyst. However, we observed in our cohort that patients with a dorsally herniated spinal cord without "cele" have a more stable clinical course.,12,16,21 We hypothesize that in the absence of a terminal cyst, cord-lipoma herniation outside the confines of the spinal canal, into the subcutaneous fat, may protect against deterioration in infancy. ${ }^{12,16,21}$ Due to the rarity of these lesions, further study is needed to better characterize this malformation.

We observed different patterns of clinical deterioration in $\mathrm{ED}$ and $\mathrm{LD} / \mathrm{S}$ groups. The development of isolated bladder dysfunction in patients in the LD/S group is suggestive of dysfunction at the sacral levels of the spinal cord, which we believe is the result of tethering on the lower spinal cord. ${ }^{25}$ In contrast, patients in the ED group often presented with multiple deficits including motor/sensory/ bowel/bladder, suggestive of more diffuse dysfunction. Given that these patients were also more likely to have more expansive lesions, we suggest that a compressive etiology is the predominant pathophysiology underlying their deterioration. It is unclear why no patients deteriorated between 18 and 30 months, but it may reflect our limited study population.

This dual etiology of deterioration may be important 

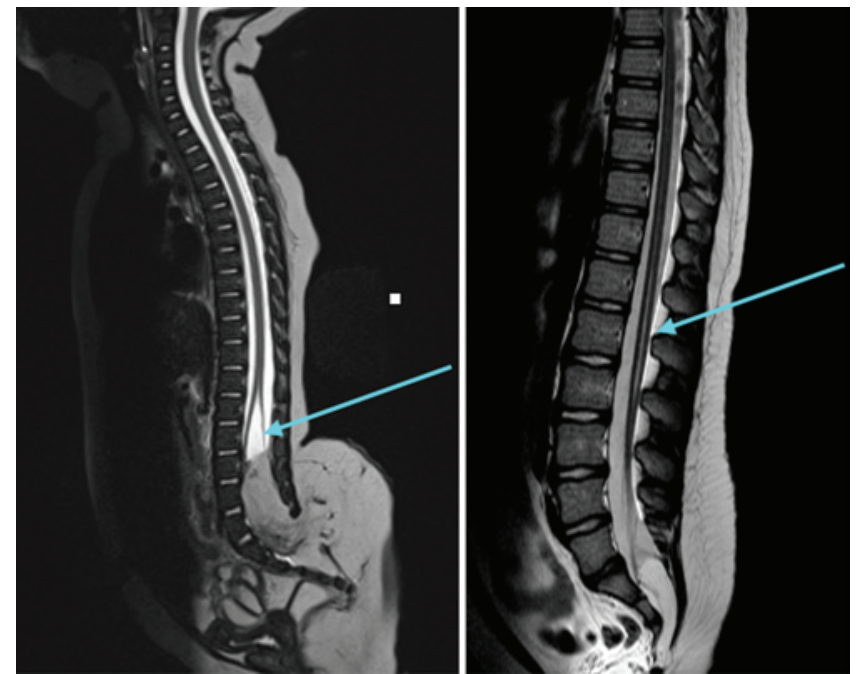

FIG. 4. Comparison of syrinx between patients with early and late deterioration. Left: Syrinx morphology typical of the ED group. The syrinx (arrow) extends rostrally from the lipoma-cord interface and expands the cord. The lipoma below is contained in an intact spinal canal. Right: Syrinx or persistent central canal (arrow) in the LD/S group does not exert mass effect. Figure is available in color online only.

when considering the goals of any surgical intervention. It suggests that in infancy, because sustained untethering is difficult to ensure, aggressive lipoma debulking, syrinx fenestration, and an expansile duraplasty should be principal goals of surgery whether safe untethering can be achieved or not. Initial or repeat detethering at a later date could be performed if necessary. ${ }^{6,13,14,22}$ This approach might address symptoms in infancy related to a direct compression and delay or avoid riskier detethering.

Similarly, prediction of clinical course allows for more personalized approaches to treatment. Initial conservative management in very young or difficult-to-assess patients may allow latent neurological worsening to occur in an irreversible fashion. Those patients with imaging features that put them into a higher-risk category for deterioration should be operated upon early, prior to deterioration, or at least have more detailed follow-up. In comparison, patients without high-risk features on imaging may be more comfortably followed clinically until they are older or become symptomatic.

We acknowledge that this study has limitations related to its small sample size and retrospective nature. The rigor of clinical monitoring provided to these patients during and after their period of nonoperative observation might not be possible in all centers. In addition, the radiographic features were determined post hoc. After speculating that neural compression from an expanding syrinx and lipoma within an intact spinal canal could result in deterioration, we assessed images and characterized the anatomical features reported. This post hoc determination of the radiographic variables could have introduced an observation bias, but would not have affected the determination of deterioration.

All MR images for study patients were reviewed independently and without concurrent access to the clini-

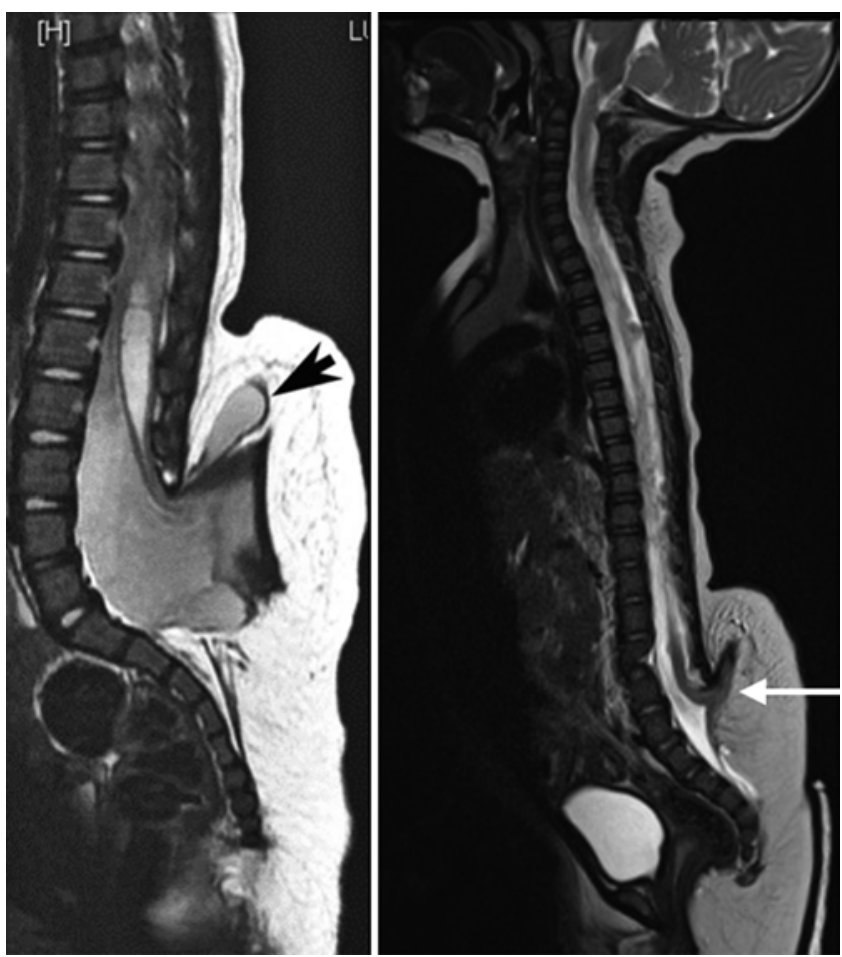

FIG. 5. Comparison of terminal myelocystocele and dorsal cord herniation. Left: Myelocystocele with dorsally situated cord in the subcutaneous lipoma. The arrow shows the "cele." There is a rostral dilated central canal but the lipoma is not contained within the intact spinal canal. Reproduced with permission from Pang D, Zovickian J, Lee JY, Moes GS, Wang KC: Terminal myelocystocele: surgical observations and theory of embryogenesis. Neurosurgery 70:1383-1405, 2012. Right: Dorsally situated extraspinal cord (arrow) with lipoma in the LD/S group.

cal outcomes. However, the reviewers were aware that an operation had been performed based on features present on postoperative studies. No formal process was used to independently evaluate these observations.

All patients underwent urodynamic testing in the 1st year and subsequently as indicated. The challenges in providing reliable urodynamic testing in infancy are well known. This unreliability may have led us to underestimate the number of patients in the ED group who showed urinary deterioration. ${ }^{1,4,20}$

This is an initial study of features that together may allow stratification of patients on the basis of their risk for early deterioration in infancy. Early deterioration would merit an early operation for decompression/untethering or at least more frequent follow-up. Our evidence suggests that patients without these risk features can be safely monitored by a comprehensive multidisciplinary program. Many of these patients at low risk will remain stable without deterioration. Additional patients are needed to validate our observations and to provide the foundation for the development of a risk-stratification tool to assist in the management of this inhomogeneous group.

\section{Conclusions}

It is possible to relate different patterns of clinical dete- 
rioration with MRI findings in patients with lumbosacral lipoma. Patients who deteriorated early in life were found to have large lipomas that filled the spinal canal, large expanding syrinxes, and displacement of neurological structures. In comparison, dorsal spinal cord herniation appears to be associated with delayed or no deterioration. These findings can be used to guide the timing of interventions and the diligence of clinical and urodynamic follow-up. Patients with features suggesting the possibility of worsening in infancy may benefit from a prophylactic intervention prior to clinical deterioration or more frequent clinical assessment. On the other hand, patients with features predictive of delayed or no worsening may avoid surgery until it is clinically indicated.

\section{Acknowledgments}

Funding for this review was provided by the Spina Bifida and Hydrocephalus Association of Canada with the grant entitled "Outcome Evaluation of a Paradigm for the Management of Lumbrosacral Lipomas" (UBC no. F14-02757).

\section{References}

1. Bauer SB, Hallett M, Khoshbin S, Lebowitz RL, Winston $\mathrm{KR}$, Gibson S, et al: Predictive value of urodynamic evaluation in newborns with myelodysplasia. JAMA 252:650-652, 1984

2. Blount JP, Tubbs RS, Okor M, Tyler-Kabara EC, Wellons JC III, Grabb PA, et al: Supraplacode spinal cord transection in paraplegic patients with myelodysplasia and repetitive symptomatic tethered spinal cord. J Neurosurg 103 (1 Suppl):36-39, 2005

3. Cochrane DD, Finley C, Kestle J, Steinbok P: The patterns of late deterioration in patients with transitional lipomyelomeningocele. Eur J Pediatr Surg 10 (Suppl 1):13-17, 2000

4. Drzewiecki BA, Bauer SB: Urodynamic testing in children: indications, technique, interpretation and significance. J Urol 186:1190-1197, 2011

5. Endoh M, Iwasaki Y, Koyanagi I, Hida K, Abe H: Spontaneous shrinkage of lumbosacral lipoma in conjunction with a general decrease in body fat: case report. Neurosurgery 43:150-152, 1998

6. Gaynor JW, Stopp C, Wypij D, Andropoulos DB, Atallah J, Atz AM, et al: Neurodevelopmental outcomes after cardiac surgery in infancy. Pediatrics 135:816-825, 2015

7. Gupta DK, Mahapatra AK: Terminal myelocystoceles: a series of 17 cases. J Neurosurg 103 (4 Suppl):344-352, 2005

8. Jansson UB, Hanson M, Hanson E, Hellström AL, Sillén U: Voiding pattern in healthy children 0 to 3 years old: a longitudinal study. J Urol 164:2050-2054, 2000

9. Jansson UB, Hanson M, Sillén U, Hellström AL: Voiding pattern and acquisition of bladder control from birth to age 6 years - a longitudinal study. J Urol 174:289-293, 2005

10. Kulkarni AV, Pierre-Kahn A, Zerah M: Conservative management of asymptomatic spinal lipomas of the conus. Neurosurgery 54:868-875, 2004

11. Kuzawa CW: Adipose tissue in human infancy and childhood: an evolutionary perspective. Am J Phys Anthropol 107 (Suppl 27):177-209, 1998

12. Lee JY, Phi JH, Kim SK, Cho BK, Wang KC: Urgent surgery is needed when cyst enlarges in terminal myelocystoceles. Childs Nerv Syst 27:2149-2153, 2011
13. McCann ME, Soriano SG: Is anesthesia bad for the newborn brain? Anesthesiol Clin 27:269-284, 2009

14. Miyamoto Y: [Influences of general anesthetics on the developing mammalian brain.] Masui 60:597-602, 2011 (Jpn)

15. Murata Y, Kanaya K, Wada H, Wada K, Shiba M, Kato Y: Reduction of caudal traction force using dural sac opening rather than spinal cord detethering for tethered cord syndrome caused by lipomyelomeningocele: a case report. Spine J 14:e1-e3, 2014

16. Pang D, Zovickian J, Lee JY, Moes GS, Wang KC: Terminal myelocystocele: surgical observations and theory of embryogenesis. Neurosurgery 70:1383-1405, 2012

17. Pang D, Zovickian J, Oviedo A: Long-term outcome of total and near-total resection of spinal cord lipomas and radical reconstruction of the neural placode, part II: outcome analysis and preoperative profiling. Neurosurgery 66:253-273, 2010

18. Pang D, Zovickian J, Oviedo A: Long-term outcome of total and near-total resection of spinal cord lipomas and radical reconstruction of the neural placode: part I-surgical technique. Neurosurgery 65:511-529, 2009

19. Pierre-Kahn A, Zerah M, Renier D, Cinalli G, Sainte-Rose C, Lellouch-Tubiana A, et al: Congenital lumbosacral lipomas. Childs Nerv Syst 13:298-335, 1997

20. Sidi AA, Dykstra DD, Gonzalez R: The value of urodynamic testing in the management of neonates with myelodysplasia: a prospective study. J Urol 135:90-93, 1986

21. Tandon V, Garg K, Mahapatra AK: Terminal myelocystocele: a series of 30 cases and review of the literature. Pediatr Neurosurg 48:229-235, 2012

22. Tiret L, Nivoche Y, Hatton F, Desmonts JM, Vourc'h G: Complications related to anaesthesia in infants and children. A prospective survey of 40240 anaesthetics. Br J Anaesth 61:263-269, 1988

23. Van Calenbergh F, Vanvolsem S, Verpoorten C, Lagae L, Casaer P, Plets C: Results after surgery for lumbosacral lipoma: the significance of early and late worsening. Childs Nerv Syst 15:439-443, 1999

24. Wykes V, Desai D, Thompson DNP: Asymptomatic lumbosacral lipomas - a natural history study. Childs Nerv Syst 28:1731-1739, 2012

25. Yamada S, Won DJ, Pezeshkpour G, Yamada BS, Yamada SM, Siddiqi J, et al: Pathophysiology of tethered cord syndrome and similar complex disorders. Neurosurg Focus 23(2):E6, 2007

\section{Disclosures}

The authors report no conflict of interest concerning the materials or methods used in this study or the findings specified in this paper.

\section{Author Contributions}

Conception and design: all authors. Acquisition of data: Tu, Hengel. Analysis and interpretation of data: all authors. Drafting the article: all authors. Critically revising the article: all authors. Reviewed submitted version of manuscript: all authors. Approved the final version of the manuscript on behalf of all authors: Cochrane. Statistical analysis: Tu, Hengel. Study supervision: Cochrane.

\section{Correspondence}

D. Douglas Cochrane, Division of Pediatric Neurosurgery, British Columbia Children's Hospital, 4480 Oak St., Rm. K3-216, Vancouver, BC V6H 3V4, Canada. email: dcochrane@cw.bc.ca. 